\title{
Amalgam Uzaylarındaki Bazı Çarpanlar ve Rölatif Tamlanış Üzerine
}

\author{
Cihan ÜNAL ${ }^{1}$ \\ ${ }^{1}$ Ölçme, Seçme ve Yerleştirme Merkezi, 06800, Bilkent/Ankara \\ e-posta: cihanunal88@gmail.com ORCIDID: http://orcid.org/0000-0002-7242-393X \\ Geliş Tarihi: 25.03.2020 Kabul Tarihi: 30.09.2020
}

Öz

Anahtar kelimeler

G, $\mu$ Haar ölçümüne sahip yerel tıkız değişmeli bir grup olsun. Bu çalışmada ilk olarak, $\left(\mathrm{L}^{\mathrm{p}}, \ell^{q}\right)(\mathrm{G})$ amalgam uzayı tanıtıldı ve bazı temel özellikleri verildi. Ayrıca, $\left(\mathrm{L}^{\mathrm{p}}, \ell^{\mathrm{q}}\right)(\mathrm{G})$ amalgam uzayının doğrusal

modül; Rölatif tamlanış; İzomorfizma bir A alt uzayı için bir A rölatif tamlanış tanımlandı ve bu tamlanışın bazı özellikleri ele alındı. Son olarak; $\operatorname{Hom}_{L^{1}(G)}\left(L^{1}(G), A\right)$ ile $A$ arasında cebirsel bir izomorfizma ve homeomorfizma olduğu ispatlandı.

\section{On Some Multipliers and the Relative Completion in Amalgam Spaces}

\begin{tabular}{cl} 
& Abstract \\
\cline { 2 - 3 } $\begin{array}{c}\text { Keywords } \\
\text { Amalgam space; }\end{array}$ & Let $\mathrm{G}$ be a locally compact abelian group with Haar measure $\mu$. First of all, in this paper, the amalgam \\
$\begin{array}{c}\text { Banach module; } \\
\text { Relative completion; }\end{array}$ & relative completion $\mathrm{A}$ for a linear subspace $\mathrm{A}$ of amalgam space $\left(\mathrm{L}^{\mathrm{p}}, \ell^{\mathrm{q}}\right)(\mathrm{G})$ is introduced and some basic properties of amalgam space are given. Moreover, a \\
Isomorphism & considered several properties of it. Finally, it is proved that there is an algebraic isomorphism and \\
& homeomorphism between $\operatorname{Hom}_{\mathrm{L}^{1}(\mathrm{G})}\left(\mathrm{L}^{1}(\mathrm{G}), \mathrm{A}\right)$ and $\mathrm{A}$.
\end{tabular}

(C) Afyon Kocatepe Üniversitesi

\section{Giriş ve Ön Bilgiler}

Çalışmanın tamamı boyunca $G, \mu$ Haar ölçümlü tıkız olmayan ve ayrık olmayan yerel tıkız Abel bir grup olarak kabul edilecektir. Yerel olarak $L^{p}$ uzayına, evrensel olarak $\ell^{q}$ uzayına ait ölçülebilir ve reel değerli fonksiyonların uzayına amalgam uzayı denir ve bu $\left(\mathrm{L}^{\mathrm{p}}, \ell^{\mathrm{q}}\right)(\mathrm{G}) \quad(1 \leq \mathrm{p}, \mathrm{q} \leq \infty)$ ile gösterilir. Holland (1975) amalgamları reel eksende araştırdı. Stewart (1979) ise bu tanımı yerel tıkız gruplar için Structure Teoremi'ni kullanarak yerel tıkız Abel gruplarına genelleştirdi. Daha kapsamlı bilgi için Fournier ve Stewart (1985) çalışmasını referans verebiliriz. Ayrıca, Quek ve Yap (1979), $L^{p}(G)$ uzayının doğrusal bir alt uzayı olan A'nın $A$ rölatif bir tamlanış tanımladı. Yazarlar $L^{1}(G)$ uzayından A uzayına giden çarpanlar uzayının cebirsel izormorfik ve homeomorfik olarak A olduğunu araştırmıştır. Ayrıca, benzer sonuçları ağırlıklı Lebesgue ve Lorentz uzayları için Duyar ve Gurkanli (2003 ve 2007) çalışmasında sağlanmıştır.

Öteleme operatörü olarak $T_{y}$, her $x \in G$ için $T_{y} f(x)=f(x-y)$ şeklinde tanımlansın. Ayrıca, her $f \in B$ ve $y \in G$ için $T_{y} f \in B$ ve $\left\|T_{y} f\right\|_{B}=\|f\|_{B}$ sağlanıyorsa $\left(B,\|.\|_{B}\right)$ ötelemeler altında güçlü değişmezdir denir. Şimdi $\left(A,\|.\|_{A}\right)$ bir Banach cebiri olsun. Ayrıca $\left(X,\|.\|_{X}\right)$ bir sol Banach A-modül olsun, yani, $X$ uzayı $A$ üzerinde cebirsel anlamda bir modül olsun ve her $a \in A$ ve $b \in X$ için $\|a \cdot b\|_{X} \leq\|a\|_{A}\|b\|_{X}$ sağlansın. Bu takdirde

$$
A X=\{a x: a \in A, x \in X\}
$$

tarafından gerilen $X^{\prime}$ in kapalı bir doğrusal alt uzayına $X$ 'in esas kısmı olarak adlandırılır ve $X_{e}$ ile gösterilir. Eğer $X_{e}=X$ ise $X^{\prime}$ e esas sol Banach $A$ - 
modülü denir (Doran ve Wichmann, 1979). Şimdi A Banach cebiri üzerinde $V$ ve $W$ iki Banach modülü alınsın. Bu takdirde $V$ üzerinden $W$ 'ye giden bir çarpan $V$ üzerinden W'ye giden bir T sınırlı doğrusal operatörüdür. Burada $a \in A$ ve $v \in V$ olmak üzere $\mathrm{T}(\mathrm{av})=\mathrm{aT}(\mathrm{v})$ olan modül çarpımı vardır. $\operatorname{Hom}_{\mathrm{A}}(\mathrm{V}, \mathrm{W})$ olarak $\mathrm{V}$ üzerinden $\mathrm{W}^{\prime}$ ye giden tüm çarpanların uzayı olarak gösterilsin. Ayrıca, $\operatorname{Hom}_{A}(V, V)=\operatorname{Hom}_{A}(V)$ olarak yazıısın.

G üzerinde bir Banach fonksiyon uzayı (kısaca BFuzayı), bir $\left(B,\|\cdot\|_{B}\right)$ Banach uzayının $L_{l o c}^{1}(G)$ uzayına sürekli olarak gömülmesine denir, yani, herhangi bir $K \subset G$ tıkız alt kümesi verildiğinde her $f \in B$ için $\left\|\mathrm{f} \chi_{\mathrm{K}}\right\|_{L^{1}} \leq \mathrm{C}_{\mathrm{K}}\|\mathrm{f}\|_{\mathrm{B}}$ olacak şekilde bir $\mathrm{C}_{\mathrm{K}}>0$ sabiti vardır. Ayrıca $L_{\text {loc }}^{p}(G)$ ile $G$ kümesinin her tıkız alt kümesine kısıtlanışların $L^{\mathfrak{p}}(G)$ uzayına ait olan fonksiyonların uzayı gösterilsin. Structure Teoreminden (Hewitt ve Ross, 1979, Teorem 24.30) $\mathrm{G}=\square^{\mathrm{a}} \times \mathrm{G}_{1}$ şeklindedir. Burada a bir negatif olmayan bir tam sayı ve $\mathrm{G}_{1}, \mathrm{H}$ açık tıkız alt grubunu içeren bir yerel tıkız Abel gruptur. Şimdi $I=[0,1)^{a} \times H$ ve $T, H$ 'nin $G_{1}$ içinde bir çaprazlama yani $G_{1}=\bigcup_{t \in T}(t+H)$ olmak üzere $J=\square^{a} \times T$ şeklinde alınsın. $\alpha \in \mathrm{J}$ için $\mathrm{I}_{\alpha}=\alpha+\mathrm{I}$ ifade edilsin ve böylece $\mathrm{G}, \mathrm{I}_{\alpha}$ rölatif tıkız kümelerin ayrık birleşimine eşittir. Tüm $\alpha$ için $\mu(I)=\mu\left(I_{\alpha}\right)=1$ olacak şekilde $\mu$ normalleştirilsin. Şimdi $\left(\mathrm{L}^{\mathrm{p}}, \ell^{\mathrm{q}}\right)(\mathrm{G})$ uzayının tanımı (Stewart, 1979) çalışmasına göre verilsin.

Tanım $1.1\left(\mathrm{~L}^{\mathrm{p}}, \ell^{\mathrm{q}}\right)(\mathrm{G})=\left(\mathrm{L}^{\mathrm{p}}, \ell^{\mathrm{q}}\right)$ şeklinde gösterilen amalgam uzayları

$$
\left\{\mathrm{f} \in \mathrm{L}_{\mathrm{loc}}^{\mathrm{p}}(\mathrm{G}):\|\mathrm{f}\|_{\mathrm{pq}}<\infty\right\}
$$

olarak ifade edilir. Burada

$$
\begin{array}{ll}
\|f\|_{p q}=\left[\sum_{\alpha \in J}\|f\|_{L^{p}\left(I_{\alpha}\right)}^{q}\right]^{\frac{1}{q}} & 1 \leq p, q<\infty \text { için } \\
\|f\|_{\infty \propto q}=\left[\sum_{\alpha \in J} \sup _{\left.x \in\right|_{\alpha}}|f(x)|^{q}\right]^{\frac{1}{a}} & p=\infty, 1 \leq q<\infty \text { için } \\
\|f\|_{p \infty}=\sup _{\alpha \in J}\|f\|_{\mathbb{P}^{(}\left(I_{\alpha}\right)} & 1 \leq p<\infty, q=\infty \text { için }
\end{array}
$$

şeklinde tanımlanır. Ayrıca amalgam uzaylarının $1<p, q<\infty$ için yansımalı Banach uzayları olduğu biliniyor.

Tanım 1.2 ((Doran ve Wichmann, 1979), (Squire, 1984)) A bir Banach cebiri olsun. Eğer

(i) Her $f, g \in A, h \in B$ için $(f \cdot g) \cdot h=f \cdot(g \cdot h)$ eşitliği sağlansın

(ii) Her $f \in A, h \in B$ için $\|f \cdot h\|_{B} \leq C\|f\|_{A}\|h\|_{B}$ olacak şekilde $C \geq 1$ sabiti olsun

koşullarını sağlayan bir $: A \times B \rightarrow B$ bilineer operatör varsa B Banach uzayına bir Banach Amodülü adı verilir.

Tanım 1.3 ((Bertrandis ve Darty, 1978), (Busby ve Smith, 1981), (Squire, 1984)) $p, q, r, s$ üsleri $\frac{1}{\mathrm{p}}+\frac{1}{\mathrm{r}}-1=\frac{1}{\mathrm{~m}} \leq 1$ ve $\frac{1}{\mathrm{q}}+\frac{1}{\mathrm{~s}}-1=\frac{1}{\mathrm{n}} \leq 1 \quad$ koşullarını sağlıyorsa

$$
\left(\mathrm{L}^{\mathrm{p}}, \ell^{\mathrm{q}}\right) *\left(\mathrm{~L}^{\mathrm{r}}, \ell^{\mathrm{s}}\right) \subset\left(\mathrm{L}^{\mathrm{m}}, \ell^{\mathrm{n}}\right)
$$

kapsaması sağlanır.

Ayrıca, eğer $f \in\left(L^{p}, \ell^{q}\right)$ ve $g \in\left(L^{r}, \ell^{s}\right)$ ise,

$$
\|f * g\|_{m n} \leq 2^{a}\|f\|_{p q}\|g\|_{r s} \quad m \neq 1 \text { için }
$$

$$
\left\|f^{*} g\right\|_{1 \mathrm{n}} \leq 2^{2 \mathrm{a}}\|\mathrm{f}\|_{1 \mathrm{q}}\|g\|_{1 \mathrm{~s}} .
$$

elde edilir.

(1) eşitsizliğinden herhangi $f \in\left(L^{p}, \ell^{q}\right)$ için

$$
\|\mathrm{f} * \mathrm{~g}\|_{\mathrm{pq}} \leq \mathrm{C}\|\mathrm{f}\|_{\mathrm{pq}}\|\mathrm{g}\|_{11}=\mathrm{C}\|\mathrm{f}\|_{\mathrm{pq}}\|\mathrm{g}\|_{1}
$$

ifadesi bulunur ve $g \in\left(\mathrm{L}^{1}, \ell^{1}\right)=\mathrm{L}^{1}$ sağlanır. Burada $\mathrm{C} \geq 1$ 'dir. $\mathrm{Bu}$ ise $\left(\mathrm{L}^{\mathrm{p}}, \ell^{\mathrm{q}}\right)$ amalgam uzayının girişim işlemine göre bir Banach $L^{1}$-modülü olduğunu gösterir (Squire, 1984). Ayrıca, $\left(\mathrm{L}^{\mathrm{p}}, \ell^{1}\right)$ amalgam uzayının $p \geq 1$ için girişim altında bir Banach cebiri olduğunu görmek kolaydır. Gerçekten, (1) eşitsizliğinden dolayı her $\mathrm{f}, \mathrm{g} \in\left(\mathrm{L}^{\mathrm{p}}, \ell^{1}\right)$ fonksiyonları için

$$
\|f * g\|_{p 1} \leq \mathrm{C}\|\mathrm{f}\|_{1}\|\mathrm{~g}\|_{p 1}=\mathrm{C}\|\mathrm{f}\|_{p 1}\|\mathrm{~g}\|_{p 1}
$$


bulunur. Böylece $\|\mathrm{f}\|_{\mathrm{p} 1}=\mathrm{C}\|\mathrm{f}\|_{\mathrm{p} 1}$ ifadesi $\left(\mathrm{L}^{\mathrm{p}}, \ell^{1}\right)$ uzayı için bir norm belirtir. Burada $\left(\mathrm{L}^{\mathrm{p}}, \ell^{1}\right) \subset \mathrm{L}^{1}$ ve $\|.\|_{1} \leq\|.\|_{p 1}$ oldukları dikkate alınmalıdır.

Teorem 1.4 (Squire, 1984) $1 \leq p, q<\infty$ olsun. Bu takdirde $\left(\mathrm{L}^{\mathrm{p}}, \ell^{\mathrm{q}}\right)$ amalgam uzayı ötelemeler altında değişmezdir, yani, her $y \in G$ ve $f \in\left(L^{p}, \ell^{q}\right)$ için $\left\|T_{y} f\right\|_{p q} \leq 2^{a}\|f\|_{p q}$ eşitsizliği sağlanır.

Teorem 1.5 (Squire, 1984) $1 \leq p, q<\infty$ olsun. $O$ halde $\mathrm{y} \rightarrow \mathrm{T}_{\mathrm{y}}$ fonksiyonu $\mathrm{G}^{\prime}$ den $\left(\mathrm{L}^{\mathrm{p}}, \ell^{\mathrm{q}}\right)$ uzayına süreklidir.

$L^{1}(G)$ uzayının bir altcebiri olan $S(G)$ aşağıdaki koşulları sağlarsa bir Segal cebiri adı verilir;

(S-1) $S(G), L^{1}(G)$ uzayında yoğundur ve $f \in S(G)$ için $T_{y} f \in S(G)$ sağlanır

(S-2) $S(G),\|.\|_{s(G)}$ normunda göre bir Banach cebiri olup her $f \in S(G), y \in G$ için $\|f\|_{S(G)}=\left\|T_{y} f\right\|_{S(G)}$ eşitliği sağlanır

(S-3) $f \in S(G)$ olsun. Bu takdirde her $\varepsilon>0$ sayısı verildiğinde tüm $y \in U$ için $\left\|T_{y} f-f\right\|_{S(G)}<\varepsilon$ olacak şekilde G'nin birim elemanının bir $U$ komşuluğu vardır.

Şimdi, $\left(L^{\mathfrak{p}}, \ell^{\mathrm{q}}\right)$ uzayının normuna denk ötelemeler altında değişmez \|\|$_{p q}^{\#}$ normuna sahip olduğu ifade edilecektir.

Teorem 1.6 ((Fournier ve Stewart, 1985), (Squire, 1984)) Bir $f$ fonksiyonunun $1 \leq p, q<\infty$ olmak üzere $\left(\mathrm{L}^{\mathrm{p}}, \ell^{\mathrm{q}}\right)$ uzayına ait olması için gerek ve yeter koşul $G$ üzerinde tanımlı $f^{\#}(x)=\|f\|_{L^{(}(x+E)}$ biçiminde tanımlı $f^{\#}$ fonksiyonunun $L^{q}(G)$ uzayına ait olmasıdır. Eğer $\|f\|_{p q}^{\#}=\left\|f^{\#}\right\|_{q} \quad$ ise, $2^{-a}\|f\|_{p q}=\|f\|_{p q}^{\#} \leq 2^{a}\|f\|_{p q}$ sağlanır ve $E$ sıfııın açık prekompakt komşuluğu olmak üzere

$$
\|f\|_{p q}^{\#}=\left[\int_{G}\|f\|_{L^{p}(x+E)}^{q} d x\right]^{\frac{1}{q}}
$$

şeklindedir. Böylece \|\|$_{p q}$ ve \|\|$_{p q}^{\#}$ normları denktirler.

Teorem 1.6'nın bir sonucu olarak aşağıdaki sonuç elde edilir.

Sonuç 1.7 Herhangi $f \in\left(L^{p}, \ell^{q}\right)$ alınsın ve $f^{\#}(x)=\|f\|_{L^{P}(x+E)}$ olsun. Bu takdirde

$$
\begin{aligned}
\left(T_{y} f\right)^{\#}(x) & =\left\|T_{y} f\right\|_{L^{p}(x+E)}=\|f\|_{L^{D}(x+y+E)} \\
& =f^{\#}(x+y)=T_{-y} f^{\#}(x)
\end{aligned}
$$

ve

$$
\left\|T_{y} f\right\|_{p q}^{\#}=\left\|\left(T_{y} f\right)^{\#}\right\|_{q}=\left\|T_{-y} f^{\#}\right\|_{q}=\left\|f^{\#}\right\|_{q}=\|f\|_{p q}^{\#}
$$

sağlanır. Böylece $\left(L^{p}, \ell^{q}\right)$ uzayı $\|\cdot\|_{p q}^{\#}$ normuna göre ötelemeler altında güçlü değişmezdir.

Teorem 1.8 (Squire, 1984) $1 \leq p<\infty$ olsun. Bu takdirde $\left(\mathrm{L}^{\mathrm{p}}, \ell^{1}\right)$ amalgam uzayı $\|.\|_{\mathrm{pq}}^{\#}$ normuna göre bir Segal cebiridir.

Tanım 1.9 Her $a \in A$ için $\lim _{\alpha} e_{\alpha} a=a$ eşitliğini sağlayan ve A değişmeli normlu cebirin bir elemanı olan $\left\{\mathrm{e}_{\alpha}\right\}$ ağı bir yaklaşık birim kısaca a.i. olarak adlandırıır.

Önerme 1.10 (Squire, 1984) $1 \leq p, q<\infty$ ve $\left\{e_{\alpha}\right\}$ ağı $\mathrm{L}^{1}$ uzayında a.i. olsun. Bu takdirde $\left\{\mathrm{e}_{\alpha}\right\},\left(\mathrm{L}^{\mathrm{p}}, \ell^{q}\right)$ uzayında da bir a.i.'dir yani her $f \in\left(L^{p}, \ell^{q}\right)$ için $\lim _{\alpha}\left\|e_{\alpha} * f-f\right\|_{p q}=0$ sağlanır.

Sonuç 1.11 (Squire, 1984) $1 \leq p, q<\infty$ olsun. Bu takdirde $\left(\mathrm{L}^{\mathrm{p}}, \ell^{\mathrm{q}}\right)$ amalgam uzayı bir esas $\mathrm{L}^{1}(\mathrm{G})$ modüldür.

\section{Temel Sonuçlar}

Tanım 2.1 A, $\left(\mathrm{L}^{\mathrm{p}}, \ell^{\mathrm{q}}\right)$ uzayının bir alt uzayı olarak aşağıdaki özellikleri sağlasın:

(i) $\left(A,\|\cdot\|_{A}\right)$ girişim işlemine göre bir Banach $L^{1}-$ modüldür ve $\|\cdot\|_{p q} \leq\|\cdot\|_{A}$ eşitsizliği sağlanır. 
(ii) Her $f \in A$ için $\lim _{\alpha}\left\|e_{\alpha}{ }^{*} f-f\right\|_{A} \rightarrow 0$ ve tüm $\alpha \in I$, bir $M>0$ sayısı için $\left\|e_{\alpha}\right\|_{1} \leq M$ olacak şekilde $L^{1}(G)$ uzayının sınırı bir $\left\{\mathrm{e}_{\alpha}\right\}$ yaklaşık birimi vardır

A uzayının rölatif tamlanış uzayı

$A=\left\{f \in\left(L^{p}, \ell^{q}\right):\right.$ Her $\alpha \in l$ için $\left.f^{*} e_{\alpha} \in A, \sup _{\alpha \in l}\left\|f^{*} e_{\alpha}\right\|_{A}<\infty\right\}$ şeklinde tanımlanır.

A uzayının $\|f\|_{A}=\sup _{\alpha \in l}\left\|f^{*} e_{\alpha}\right\|_{A}$ şeklinde tanımlı $\|\cdot\|_{A}$ normuna göre bir normlu uzay olduğunu görmek kolaydır.

Önerme 2.2 A, Tanım 2.1'deki gibi olsun. Bu takdirde

(i) $f \in A$ ve $g \in L^{1}(G)$ olsun. $O$ halde $f^{*} g \in A$ ve

$$
\left\|f^{*} g\right\|_{A} \leq\|f\|_{A}\|g\|_{1}
$$

sağlanır.

(ii) $f \in A$ olsun. $O$ halde $\|f\|_{A} \leq M\|f\|_{A}$ ve $\|f\|_{A} \leq\|f\|_{A}$ sağlanır. Bu ise $\|\cdot\|_{A}$ ve $\|\cdot\|_{A}$ normlarının $A$ üzerinde denk olduklarını gösterir.

(iii) A uzayı A uzayının kapalı bir alt uzayıdır.

İspat (Duyar ve Gurkanli, 2007) çalışmasındaki Proposition 2.2 yardımıyla istenen ifadeler elde edilir.

Önerme 2.3 A uzayının tanımı yaklaşık birimin seçimine bağlı değildir.

İspat (Duyar ve Gurkanli, 2007) çalışmasındaki Proposition 2.3 dikkate alınırsa istenen elde edilir.

Teorem 2.4 (Rieffel, 1967) A yaklaşık birime sahip bir Banach cebiri ve $\mathrm{W}$ ise yansımalı bir Banach uzayı, esas A-modül olsun. $\mathrm{Bu}$ takdirde $\operatorname{Hom}_{A}(A, W) \cong W$ elde edilir.

Teorem $2.51<p, q<\infty$ olsun. $O$ halde, $\operatorname{Hom}_{L^{1}(G)}\left(L^{1}(G),\left(L^{p}, \ell^{q}\right)\right)$ uzayı ile $\left(L^{p}, \ell^{q}\right)$ uzayı arasında izometrik modül izomorfizması vardır. Bu ise $\operatorname{Hom}_{\mathrm{L}^{1}(\mathrm{G})}\left(\mathrm{L}^{1}(\mathrm{G}),\left(\mathrm{L}^{\mathrm{p}}, \ell^{\mathrm{q}}\right)\right) \cong\left(\mathrm{L}^{\mathrm{p}}, \ell^{\mathrm{q}}\right) \quad$ olduğunu gösterir.
İspat $L^{1}(G)$ uzayının yaklaşık birimli bir Banach cebiri olduğu biliniyor. Yine $\left(L^{p}, \ell^{q}\right)$ amalgam uzayı esas $L^{1}(G)$-modül olduğundan Teorem 2.4 kullanılarak $\operatorname{Hom}_{\mathrm{L}^{1}(\mathrm{G})}\left(\mathrm{L}^{1}(\mathrm{G}),\left(\mathrm{L}^{\mathrm{p}}, \ell^{\mathrm{q}}\right)\right) \cong\left(\mathrm{L}^{\mathrm{p}}, \ell^{\mathrm{q}}\right)$ elde edilir.

Teorem $2.61 \leq \mathrm{p}, \mathrm{q}<\infty$ için $\left(\mathrm{L}^{\mathrm{p}}, \ell^{\mathrm{q}}\right)$ amalgam uzayının doğrusal bir alt uzayı olan A, Tanım 2.1'deki (i) ve (ii) koşullarını sağlasın. $O$ halde $\operatorname{Hom}_{\mathrm{L}^{1}(G)}\left(\mathrm{L}^{1}(\mathrm{G}), \mathrm{A}\right)$ ve A uzayları cebirsel olarak izomorfik ve homeomorfiktir.

İspat $f \in A$ fonksiyonu alınsın. Şimdi her $g \in L^{1}(G)$ için $T_{f}(g)=f^{*} g$ biçiminde tanımlı bir $T_{f}: L^{1}(G) \rightarrow A$ fonksiyonu tanımlansın. Şimdi $\mathrm{T}_{\mathrm{f}} \in \operatorname{Hom}_{\mathrm{L}^{1}(\mathrm{G})}\left(\mathrm{L}^{1}(\mathrm{G}), \mathrm{A}\right)$ olduğu ve $\left\|\mathrm{T}_{\mathrm{f}}\right\|, \quad\|f\|_{A}$ normlarının denk olduğu gösterilecektir. Önerme 2.2'den

$$
\begin{aligned}
& \left\|f * g * e_{i}-f^{*} g^{*} e_{j}\right\|_{A} \\
& \leq\left\|f * g * e_{i}-f^{*} g^{*} e_{j}\right\|_{A} \leq\|f\|_{A}\left\|g * e_{i}-g+g-g^{*} e_{j}\right\|_{1} \\
& \leq\|f\|_{A}\left(\left\|g * e_{i}-g\right\|_{1}+\left\|g-g^{*} e_{j}\right\|_{1}\right) .
\end{aligned}
$$

elde edilir. $\left\{\mathrm{e}_{\alpha}\right\}, \mathrm{L}^{1}(\mathrm{G})$ uzayında bir yaklaşık birim olduğundan $\left\{f^{*} \mathrm{~g}^{*} \mathrm{e}_{\mathrm{i}}\right\}_{\mathrm{i} \in \mathrm{l}}, \mathrm{A}^{\prime} \mathrm{da}$ bir Cauchy ağı olup $A^{\prime} d a$ bir $h$ fonksiyonuna yakınsar. Buradan $f^{*} g \in A$ sağlanır ve

$$
\begin{aligned}
\left\|f^{*} g-h\right\|_{A} & \leq\left\|f^{*} g-f^{*} g^{*} e_{\alpha}\right\|_{A}+\left\|f^{*} g^{*} e_{\alpha}-h\right\|_{A} \\
& \leq\|f\|_{A}\left\|g-g^{*} e_{\alpha}\right\|_{1}+M\left\|f^{*} g^{*} e_{\alpha}-h\right\|_{A} \rightarrow 0
\end{aligned}
$$

elde edilir. Böylece $T_{f}(g)=f^{*} g=h \in A$ sağlanır. $O$ halde, $T_{f}: L^{1}(G) \rightarrow A$ fonksiyonu vardır. Önerme 2.2'den

$$
\begin{aligned}
\left\|T_{f}\right\| & =\sup _{g \neq 0} \frac{\left\|T_{f}(g)\right\|_{A}}{\|g\|_{1}}=\sup _{g \neq 0} \frac{\|f * g\|_{A}}{\|g\|_{1}} \\
& \leq \sup _{g \neq 0} \frac{\|f * g\|_{A}}{\|g\|_{1}} \leq \sup _{g \neq 0} \frac{\|f\|_{A}\|g\|_{1}}{\|g\|_{1}}=\|f\|_{A}
\end{aligned}
$$

sağlanır. $\mathrm{Bu}$ ise $\mathrm{T}_{\mathrm{f}}: \mathrm{L}^{1}(\mathrm{G}) \rightarrow \mathrm{A}$ fonksiyonunun sürekli olduğunu gösterir. Yine bu $T_{f}$ fonksiyonunun sürekli bir modül homomorfizması 
olduğunu ispatlamak kolaydır. Tanım 2.1 göz önüne alınırsa

$$
\begin{aligned}
\left\|T_{f}\right\| & =\sup _{g \neq 0} \frac{\left\|T_{f}(g)\right\|_{A}}{\|g\|_{1}}=\sup _{g \neq 0} \frac{\|f * g\|_{A}}{\|g\|_{1}} \geq \sup _{\alpha \in 1} \frac{\left\|f^{*} e_{\alpha}\right\|_{A}}{\left\|e_{\alpha}\right\|_{1}} \\
& \geq \sup _{\alpha \in 1} \frac{\left\|f^{*} e_{\alpha}\right\|_{A}}{M}=\frac{1}{M}\|f\|_{A}
\end{aligned}
$$

elde edilir.

Diğer taraftan, $T \in \operatorname{Hom}_{\mathrm{L}^{1}(\mathrm{G})}\left(\mathrm{L}^{1}(\mathrm{G}), \mathrm{A}\right)$ olduğu kabul edilsin. Yine $A \subset\left(L^{p}, \ell^{q}\right)$ kapsaması ve $\|\cdot\|_{p q} \leq\|\cdot\|_{A}$ eşitsizliğinden, her $g \in L^{1}(G)$ için

$$
\|\mathrm{T}(\mathrm{g})\|_{\mathrm{pq}} \leq\|\mathrm{T}(\mathrm{g})\|_{\mathrm{A}} \leq\|\mathrm{T}\|\|\mathrm{g}\|_{1}
$$

sağlanır. $O$ halde $T \in \operatorname{Hom}_{L^{1}(G)}\left(L^{1}(G),\left(L^{p}, \ell^{q}\right)\right)$ olarak bulunur. Teorem 2.5 ve (Rieffel, 1967) çalışmasındaki Theorem 4.5 kullanııısa, her $g \in L^{1}(G)$ fonksiyonu için $T(g)=f * g$ olacak şekilde bir $\mathrm{f} \in\left(\mathrm{L}^{\mathrm{p}}, \ell^{q}\right)$ fonksiyonu vardır. Tanım 2.1'den

$$
\begin{aligned}
& \|f\|_{A}=\sup _{\alpha \in 1}\left\|f * e_{\alpha}\right\|_{A}=\operatorname{Msup} \frac{\left\|f * e_{\alpha \in l}\right\|_{A}}{M} \\
& \leq \operatorname{Msup} \frac{\left\|\mathrm{f}^{*} \mathrm{e}_{\alpha}\right\|_{\mathrm{A}}}{\left\|\mathrm{e}_{\alpha}\right\|_{1}} \leq \operatorname{Msup} \frac{\left\|\mathrm{f}^{*} \mathrm{~g}\right\|_{\mathrm{A}}}{\|\mathrm{g}\|_{1}} \\
& =\underset{g \neq 0}{\operatorname{Mup}} \frac{\|\mathrm{T}(\mathrm{g})\|_{\mathrm{A}}}{\|\mathrm{g}\|_{1}}=\mathrm{M}\|\mathrm{T}\|<\infty
\end{aligned}
$$

olup $\mathrm{f} \in \mathrm{A}$ elde edilir. Böylece herbir $T \in \operatorname{Hom}_{L^{1}(G)}\left(L^{1}(G), A\right)$, bazı $f \in A$ fonksiyonları için $\left\|T_{f}\right\| \leq\|f\|_{A}$ ve $\|f\|_{A} \leq M\left\|T_{f}\right\|$ ifadelerini sağlayan $T_{f}$ 'nin bir formudur. Böylece $f \rightarrow T_{f}$ fonksiyonu cebirsel bir izomorfizma ve homeomorfizmadır.

\section{Kaynaklar}

Bertrandis, J.P., Darty, C. ve Dupuis, C. 1978. Unions et intersections d'espaces $L^{p}$ invariantes par translation ou convolution. Ann Inst Fourier Grenoble, 28(2), 5384.

Busby, R.C. ve Smith, H.A. 1981. Product-convolution operators and mixed-norm spaces. Trans Amer Math Soc., 263(2), 309-341.

Doran, R.S. ve Wichmann, J. 1979. Approximate Identities and Factorization in Banach Modules.
Lecture Notes in Mathematics XII, Springer-Verlag, 312.

Duyar, C. ve Gurkanli, A.T. 2003. Multipliers and relative completion in weighted Lorentz spaces. Acta Math Sci, 23B-4, 467-476.

Duyar, C. ve Gurkanli, A.T. 2007. Multipliers and the relative completion in $\mathrm{L}_{\omega}^{\mathrm{p}}(\mathrm{G})$. Turk J Math., 31, 181191.

Fournier, J.J. ve Stewart, J. 1985. Amalgams of $\mathrm{L}^{\mathrm{p}}$ and $\ell^{q}$. Bull Amer. Math. Soc., 13(1), 1-21.

Hewitt, E. ve Ross, K.A. 1979. Abstract Harmonic Analysis v. I, II. Berlin-Heidelberg-New York, SpringerVerlag, 774.

Holland, F. 1975. Harmonic analysis on amalgams of $L^{p}$ and $\ell^{q}$. J. London Math. Soc., 2(10), 295-305.

Quek, T.S. ve Yap, L.H. 1979. Multipliers from $L^{1}(G)$ to a Lipschitz space. J. Math. Anal. Appl., 69, 531-579.

Rieffel, H. 1967. Induced Banach representation of Banach algebras and locally compact groups. J. Funct. Anal., 1, 443-491.

Squire, M.L.T. 1984. Amalgams of $L^{p}$ and $\ell^{q}$. PhD Thesis (Doktora), McMaster University.

Stewart, J. 1979. Fourier transforms of unbounded measures. Canad. J. Math., 31(6), 1281-1292. 